\title{
Differences in the quality of life of two groups of drug users
}

\author{
Siobhan Rooney, Aideen Freyne, Gabrielle Kelly, John O’Connor
}

Ir J Psych Med 2002; 19(2): 55-59

\begin{abstract}
Objectives: The aim of this study was to compare aspects of the quality of life of drug users on a methadone maintenance programme to drug users on a harm minimisation programme.

Method: Thirty-six clients attending the harm minimisation programme in the National Drug Treatment Centre, Dublin, were matched for age and sex to 36 clients on the methadone maintenance programme. All were interviewed with the SF-36 Health Survey Questionnaire to measure health related quality of life and with the Hospital Anxiety and Depression Scale (HADs) to measure psychological morbidity.

Results: More clients from the harm minimisation programme had previous psychiatric problems than clients on the methadone maintenance programme, with an odds ratio of $4.3 \mathrm{CI}(1.2,15.2)$. On the HADs, clients on the methadone maintenance programme had significantly lower depression scores than clients on the harm minimisation programme. In addition more clients on the harm minimisation programme were severely depressed than clients on the methadone maintenance programme. On the UK SF-36 Scale, clients on the harm minimisation programme perceived a significantly greater deterioration in 'change in health' over the previous year than clients on the methadone maintenance programme.
\end{abstract}

Conclusions: Although clients on a methadone maintenance programme had an improved perception of their quality of life in relation to psychological and overall health function from the previous year, compared to clients on a harm minimisation programme, there still existed varying degrees of psychopathology in both groups which need to be considered when providing future services for drug users.

Key words: Quality of life; Methadone maintenance programme; Harm minimisation programme.

\section{Introduction}

Health, as defined by the World Health Organisation, is 'a state of complete physical, mental and social well-being

\footnotetext{
*Siobhan Rooney, Senior Registrar in Substance Misuse, National Drug Treatment Centre, Pearse St, Dublin 2, Ireland. Aideen Freyne, Consultant Psychiatrist, St Vincent's Hospital, Elm Park, Dublin 4, Ireland.

Gabrielle Kelly, Department of Statistics, University College Dublin, Belfield, Dublin 4, Ireland.

John O’Connor, Consultant Psychiatrist in Substance Misuse, National Drug Treatment Centre, Pearse Street, Dublin 2, Ireland.

*Correspondence

SUBMITTED: OCTOBER 16, 2000. ACCEPTED: FEBRUARY 23, 2002.
}

and not merely the absence of disease or infirmity'. ${ }^{1}$ Patients' perceptions of their level of functioning in these areas provides a basis for estimating their perceived quality of life. Of particular influence in defining and measuring components of 'quality of life' was the work carried out by the Rand Corporation in the Health Insurance experiment and later in the Medical Outcomes Study. ${ }^{2,3}$ They developed a self report questionnaire, the Short Form 36 (SF-36) to measure health status and quality of life.

Although there has been much interest in assessing the quality of life of patients with specific illnesses and diseases, there have been few studies that assess the quality of life of opiate drug users. Torrens et al in a prospective three year study found, through the use of the Nottingham Health Profile, that opioid users, when treated with a methadone maintenance programme for a 12 month period, experienced an early and substantial improvement in health related quality of life. ${ }^{4}$ The most significant improvements were within the first to third month of treatment. The authors suggested that measuring changes in the health related quality of life can constitute a measurement of treatment effectiveness and can be used to evaluate drug treatment programmes. Ryan et al assessed health status at entry to a methadone maintenance treatment using the SF36 health survey questionnaire and found that scores for heroin users were significantly worse for physical and psychological health than for the general population. ${ }^{5}$ Scores for psychological functioning were most similar to clinically depressed patients while self-perceived general health was equivalent to patients who had a severe medical illness.

Marsden et al explored the prevalence of psychiatric symptoms amongst clients seeking treatment for drug dependency based on data obtained from the National Treatment Outcome Research Study. ${ }^{6}$ They found a 'conditional' relationship between psychiatric symptoms and substance use. For primary opiate drug users without polydrug use, their drug intake did not correlate with psychiatric symptoms. However, when opiate use is associated with polydrug use with stimulants and benzodiazepines or alcohol use, then the severity of drug use was found to be related to psychiatric symptoms.

The Epidemiologic Catchment Area study showed that $53 \%$ of drug users had a mental disorder, with anxiety disorders being the most common diagnosis, accounting for $28 \%$ of psychiatric conditions, followed by affective disorders at $26 \% .^{7}$ Rounsaville et al found that $70 \%$ of drug users had an associated psychiatric diagnosis to drug dependency. Elevated scores for measures of depression, anxiety and antisocial personality were common in heroin using populations. ${ }^{8}$ In an Irish population of drug users on methadone maintenance, $83 \%$ exhibited some level of depression, $27 \%$ scored in the moderately depressed range 
and $56 \%$ scored in the severely depressed range of the Beck Depression Inventory. ${ }^{9}$

The aim of this study was to assess the perceived quality of life and psychological morbidity of clients on a harm minimisation programme and to compare results to clients attending a methadone maintenance drug treatment programme.

\section{Method}

All clients attending a harm minimisation programme in the National Drug Treatment Centre, Dublin, were requested to take part in the study. The programme consisted of a needle exchange programme, $20 \mathrm{mg}$ of methadone daily, and access to medical personnel. These clients were matched for age and sex to clients attending a methadone maintenance programme and written consent was obtained from all who took part in the study. Data regarding the demographic characteristics of both groups and the amount, duration and frequency of present and past drug use was collected. Information was obtained from clients about past psychiatric histories and about a range of subjective psychological experiences that they had had in the past and specifically in the previous 30 days. These included whether they had experienced depression, anxiety, difficulties in concentration, visual hallucinations, suicidal ideation or had engaged in a suicidal attempt. Specific scales were used in the study to measure psychological, physical and social function.

Scales used

The SF-36 general health questionnaire is a generic measure of health status and quality of life. It is a 36 item questionnaire which evaluates individuals' perceptions of their performance in eight dimensions of daily life, corresponding to three aspects of health, ie. functional status, well-being and 'overall evaluation of health'. The four scales measuring functional status are physical functioning (10 items), role limitations due to physical problems (four items), role limitations due to emotional problems (three items) and social functioning (two items).

'Wellbeing' has three scales: mental health (five items), energy/vitality (four items) and bodily pain (two items). The overall evaluation of health is obtained from a person's perception of their general health (five items). There is a further unscaled single item asking respondents about health change over the past year. For each variable, item scores are summed and transformed to give eight scores with a 0-100 scale. Higher scores indicate better function. The SF-36 has a US and a UK version and has been shown to be a valid and reliable instrument for measuring overall health status. ${ }^{10-12}$ Recently, normative values for an Irish population have been presented and the US version of the SF-36 was found to have acceptable consistency and validity. ${ }^{13}$ Permission for use of the UK version of the SF-36 was obtained for this study. ${ }^{14}$

The Hospital Anxiety and Depression Scale measures states of anxiety and depression. This is a self-assessment scale which has been found to be a reliable instrument for detecting states and severity of depression and anxiety in the setting of a hospital medical outpatient clinic. A score of 0-7 is interpreted as 'normal', 8-10 as 'mild', 11-14 as 'moderate' and 15-21 as 'severe' levels of anxiety or depression. ${ }^{15}$

\section{Statistical analysis}

The unit of analysis was the matched pair and there
Table 1: Demographic characteristics

Methadone

maintenance

programme

Harm

minimisation

programme

Sex $\mathbf{M}: \mathbf{F}$

$25(69 \%): 11(31 \%)$

$25(69 \%): 11(31 \%)$

\section{Marital Status}

Single

Married/co-habiting

$23(63.9 \%)$

$18(50 \%)$

Separated/divorced

$11(30.5 \%)$

$13(36.1 \%)$

$2(5.6 \%)$

$4(11.1 \%)$

Widowed

$0 \%$

$1(2.8 \%)$

\section{*Accommodation}

Home

$14(38.9 \%)$

Own house/flat

11 (30.5\%)

Rented

$23(63.9 \%)$

$17(47.2 \%)$

Hostel

$1(2.8 \%)$

$5(13.9 \%)$

*Employment

$10(27.8 \%)$

$3(8.3 \%)$

Level of Education

\begin{tabular}{lcc} 
Primary & $8(22 \%)$ & $13(36 \%)$ \\
Secondary & $28(78 \%)$ & $19(53 \%)$ \\
Higher Education & $0(0 \%)$ & $4(11 \%)$ \\
${ }^{*} p<0.05$ & & \\
\hline
\end{tabular}

were 36 pairs, each consisting of a client from the harm minimisation programme and a client matched for age and sex from the methadone maintenance programme. The differences in the two programmes were compared and subjected to appropriate tests of statistical analysis. For categorical variables with two categories the results were assessed using McNemar's test. The results of the test, the odds ratio (OR) and associated $95 \%$ confidence interval are reported. For some variables the estimated OR is infinite (OR infinite). This happens when there are no case/control pairs in which the control had been exposed to the risk factor and the case had not. In such cases, we have simply noted that the OR could not be reliably estimated. For categorical variables with more than two categories an extension of McNemar's test - a symmetry test was used. ${ }^{16}$ If in addition the categories were ordered then a conditional symmetry test was used. For continuous variables comparisons were made using the Wilcoxon signed rank test.

\section{Results}

There were $25(69 \%)$ males from each programme and the average age was 32 years. There was no difference in marital status between the two groups. Significantly more clients on the methadone maintenance programme were living in their own accommodation while more on the harm minimisation programme were living in the family home (symmetry test $\mathrm{p}=0.001$ ). Significantly more clients on the methadone maintenance programme were employed (McNemar's test $\mathrm{p}=0.05$, OR 4.5 CI $(1.0,14.6)$ ). There were no significant differences between the two 


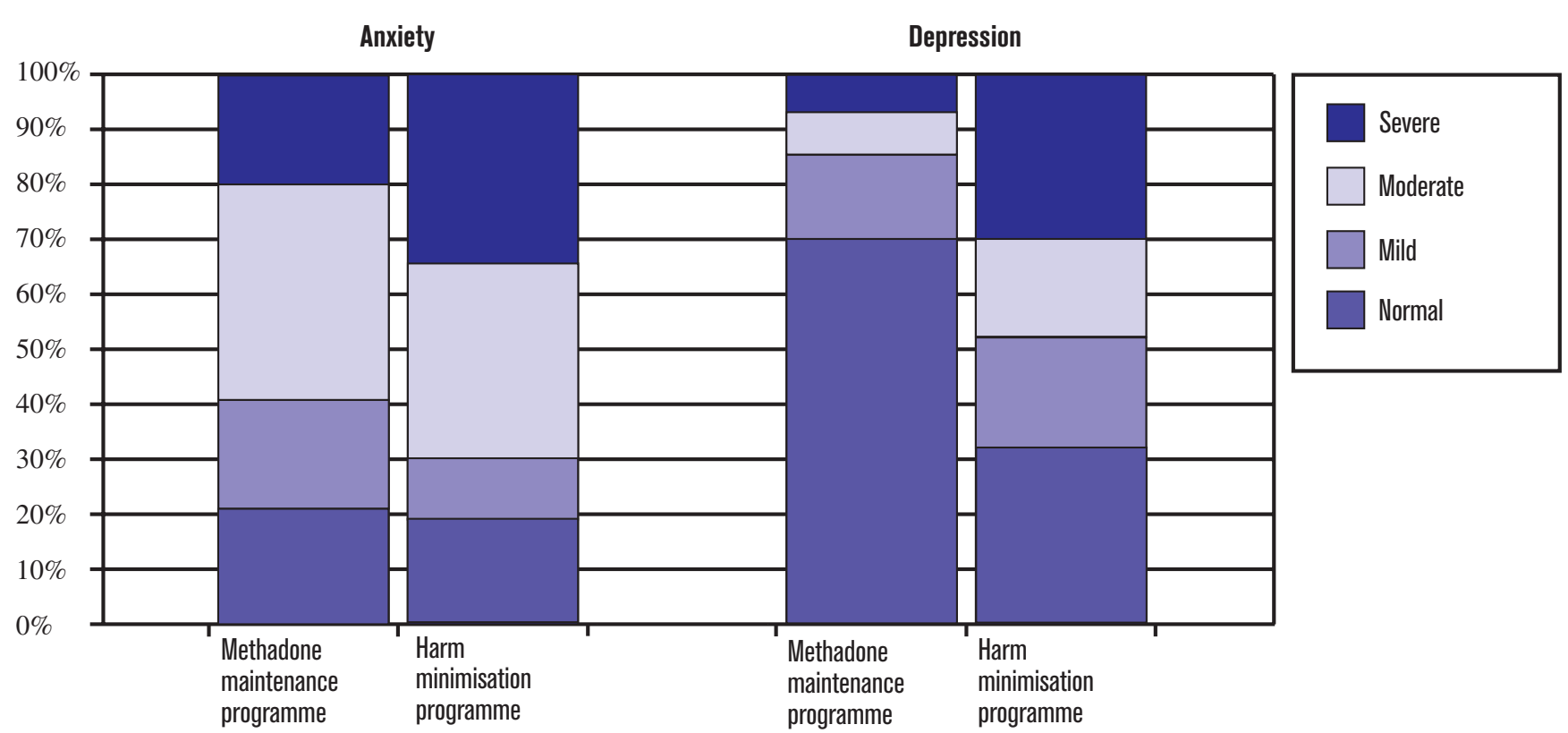

groups in terms of highest level of education obtained (see Table 1).

History of drug use

There was no difference between the two groups with regard to age of first use of specific drugs and alcohol. The main difference between the two groups was that cocaine was used significantly longer by clients on the harm minimisation programme, while use of analgesics was more common in the methadone maintenance programme. Cocaine was used for 4.4 years longer by clients on the harm minimisation programme compared to clients on the methadone maintenance programme, $\mathrm{p}=0.05$. Significantly more on the methadone maintenance programme used analgesics, $(\mathrm{p}=0.001$, OR $10.5, \mathrm{CI}(2.5,44.8))$.

There was a trend for the methadone maintenance clients to have used alcohol longer than the harm minimisation clients, the difference being 2.06 years, $\mathrm{p}=0.07$. Significantly more in the harm minimisation programme had used heroin $(\mathrm{p}=0.002$, OR $24, \mathrm{CI}(3.2,177.4))$ and benzodiazepines ( $\mathrm{p}=0.000$, OR infinite - see explanation in the methods section) in the previous 30 days prior to the interview. Significantly more clients on the harm minimisation programme had injected in general, $(p=0.000, O R$ infinite), had injected within the last six months ( $\mathrm{p}=$ 0.002, OR 6.7, $\mathrm{CI}(2.0,22.4))$ and within the previous 30 days, $(\mathrm{p}=0.000$, OR infinite). There were no significant differences in the numbers who shared needles in both groups. Clients from both groups had a mean age of first injecting at 21 years.

Clients on the harm minimisation programme had had more past treatments for drug misuse, an average of 2.7 times more compared to clients on the methadone maintenance programme, $(p=0.06)$. In particular, they had significantly more outpatient methadone stabilisation programmes, $(\mathrm{p}=0.02$, OR 4.3, $\mathrm{CI}(1.2,15.2)$.

Clients on the harm minimisation programme spent on average per month $£ 1,200$ ( $€ 1,524)$ on drugs compared to $£ 16$ (€20) for clients on the methadone maintenance programme, $\mathrm{p}=0.0001$. The amounts ranged from $£ 0$ $£ 10,000(€ 1,2697)$ per month.
Psychological health

A total of $22(61 \%)$ on the harm minimisation programme and $15(41.7 \%)$ on the methadone maintenance programme stated they had had previous psychological or emotional problems. More clients on the harm minimisation programme stated they had a previous psychiatric history, $(\mathrm{p}=0.02$, OR $4.3 \mathrm{CI}(1.2,15.2))$. There were no differences between the two groups in the number of past admissions to a psychiatric unit, but significantly more in the harm minimisation programme had attended and received treatment in a psychiatric outpatients department, $(\mathrm{p}=0.01$, OR 12.0, CI $(1.6,92.3))$.

Significantly more in the harm minimisation programme reported suffering with depression $(\mathrm{p}=0.007$, OR 7.5, $\mathrm{CI}(1.7,32.8))$ and specifically within the previous 30 days $(\mathrm{p}=0.03, \mathrm{OR} 3.0, \mathrm{CI}(1.1,8.3))$. Evidence of greater levels of drug intoxication experienced by the harm minimisation group is indicated by significantly more reporting difficulty in understanding, concentrating and remembering in general $(\mathrm{p}=0.01$, OR $5, \mathrm{CI}(1.4,17.3))$, but specifically in the past 30 days $(\mathrm{p}=0.01$, OR $4.7, \mathrm{CI}(1.3,16.2))$ and significantly more having experienced visual hallucinations $(\mathrm{p}=0.000, \mathrm{OR}$ infinite $)$. This group reported significantly more suicidal ideation $(\mathrm{p}=0.03$, OR $3, \mathrm{CI}(1.1,8.3))$ but there were no significant differences between the two groups in the number of parasuicide attempts, (mean number was one). There were no differences in the subjective experience of anxiety reported by the two groups.

On the HADs, both groups showed various degrees of anxiety, but there were no significant differences in the scores between the two groups. However, in a comparison of the depression scores from the HADs, the mean score for clients on the harm minimisation programme was 10.8 , significantly higher than the mean score of $6.4(\mathrm{p}=$ 0.00003 ) for clients on the methadone maintenance programme. In addition, the harm minimisation programme had significantly more clients registering a score for severe depression, $(\mathrm{p}=0.03$, OR 5.5, CI $(1.2,24.8))$. Twenty-four clients $(66.7 \%)$ on the harm minimisation programme registered some level of depres- 


\begin{tabular}{l}
\hline Table 2: Characteristics of attenders on harm minimisation programme \\
\hline - Numbers living at home with family \\
- Unemployed \\
- Severity of drug use \\
- Use of heroin, cocaine and benzodiazepines \\
- Number injecting \\
- Number of past treatments for drug use \\
- Monthly expenditure on drugs \\
- Psychological morbidity \\
- Perceived deterioration of health in the previous year
\end{tabular}

sion with $11(31 \%)$ severely depressed. Eleven clients $(31 \%)$ on the methadone maintenance programme had some level of depression and $2(5.1 \%)$ were severely depressed, (see Figure 1).

With regard to analysis of the UK SF-36 Scale, no differences were found in the scores between the two groups in the dimensions of physical functioning, role limitation due to physical or emotional problems, social functioning, mental health, energy and vitality, bodily pain and general health perception. Perceived 'change in health' from the previous year showed a large difference indicating a greater perceived deterioration of health for clients on the harm minimisation programme and a perceived improvement in health from the previous year for clients on the methadone maintenance programme $(\mathrm{p}=0.003)$.

\section{Discussion}

This study has methodological limitations as it offers only a snap-shot comparison of two service modalities rather than a prospective analysis over time between the two treatment programmes. Although the SF-36 has been found to be a reliable and valid instrument for measuring the quality of life in the US, UK and more recently in Ireland, it is not known if it is transferable to an Irish population of drug users. Irish drug users may have a different perception of quality of life which may be very culturally specific. The other limitation of this study is the selection of patients. A harm minimisation programme by its nature will have more chaotic clients and therefore the differences noted between the two programmes are not a measure of treatment effectiveness but of client selection, and as such, the findings need to be considered in light of these limitations.

Attenders on the harm minimisation programme were less self sufficient than clients on the methadone maintenance programme as indicated by a greater dependence on families for accommodation and a lower employment rate. They had a more severe pattern of drug use as evidenced by a larger current use of a number of drugs such as heroin, benzodiazepines and cocaine, symptoms of drug intoxication, more past treatments for drug dependency and a higher monthly expenditure on drugs. They were engaged in more risk behaviour as indicated by a greater intravenous use of drugs, both in the past and currently (see Table 2).

Clients on the harm minimisation programme had used cocaine significantly longer than clients on the methadone maintenance programme. This drug has mood alleviating properties and the longer use may be an attempt to self medicate and relieve depressive symptoms that can arise secondary to drug misuse. Alternatively, a greater vulnerability to suffering depressive symptoms may have been a primary contributing factor to taking drugs initially. Schottenfeld, in a review, summarised how cocaine users had a high rate of psychopathology for anxiety and affective disorders, attention deficit disorders and personality disorders. ${ }^{17}$

The methadone maintenance group had a higher rate of use of alcohol and analgesics. Ryan et al found that a greater use of alcohol in heroin users at entry to a methadone maintenance programme was associated with higher physical functioning and vitality but poorer social functioning. ${ }^{5}$ The reasons for the greater use of analgesics by this group is unclear, but it may be related to the degree of heroin dependency and an attempt to self medicate to treat opioid withdrawal symptoms. The use of analgesics may also identify a group of drug users who are more likely to seek treatment for their drug use because of the experience of distressing withdrawal symptoms in contrast to drug users who have adjusted to their life-style whereby physical symptoms are no longer intrusive.

Both groups differed in terms of degree of psychological symptoms. More clients on the harm minimisation programme had past psychological problems or psychiatric history with more having attended psychiatric outpatient services. More reported experiencing depression and suicidal ideation in their lives. This was confirmed by scores on the Hospital Anxiety and Depression Scale whereby, clients on the harm minimisation programme were more severely depressed than clients on the methadone maintenance programme. These results indicate a high level of psychological morbidity and suicidal risk in a group of drug users not in treatment. Although clients on the methadone maintenance programme had lower levels of depression, psychopathology still existed as $31 \%$ were depressed and $39 \%$ had had a previous suicidal attempt despite being in treatment. The evidence of psychopathology in drug users on methadone maintenance has been outlined in previous studies ${ }^{9,18,19}$ and how a failure to address psychiatric co-morbidity and substance misuse can lead to poorer treatment outcomes. ${ }^{20}$

Although no significant differences were found between the two groups in the eight dimensions of functioning on the SF-36, this may have been as a result of the small numbers involved in the study. However, what was relevant was the greater deterioration in health over the previous year perceived by the harm minimisation group. The greater severity of depressive symptoms in this group may have influenced this perception.

Predictors of psychiatric symptoms were found by Marsden et al to be related to severity of drug use, severe physical health symptoms, previous psychiatric treatment and conflict in personal relationships. ${ }^{6}$ In this study, similar risk factors for psychological morbidity were identified in clients on the harm minimisation programme. They had a more severe pattern of drug use, polydrug use, greater psychological morbidity, previous attendance at psychiatric clinics and a greater perception of a deterioration in their health than clients in treatment on methadone maintenance programmes.

From studies there is accumulating evidence of an association of suicidal behaviour and substance misuse with associated increased risk of attempted and completed suicide. ${ }^{21-25}$ Substance misuse is associated with a seven fold increase in the risk of attempted suicide, ${ }^{24}$ and a 15 fold 
increase in the risk of completed suicide. ${ }^{25}$ The increased psychological morbidity and suicidal risk in drug users has implications for the development of services and treatment programmes, the provision of educative programmes and in the planning of suicide preventative strategies.

\section{Implications for services}

This study highlights differences in perceived quality of life and psychological morbidity between drug users on a methadone maintenance programme and those on a harm minimisation programme. How best psychological/psychiatric treatment should be provided remains a source of debate, be it through the development of a specialist dual diagnosis service, or through the facilitation of liaison and networking between substance misuse services, mental health services primary care, different health, social services and voluntary sector agencies.

Hall and Farrell outlined the need for improvements in the abilities of staff in addiction services to recognise comorbid psychiatric illnesses, and promoted the development of models of joint care through greater liaison between the addiction and mental health services. ${ }^{22}$ Marsden et al emphasised the importance of a full psychiatric assessment to identify clinically significant disorders and suicidality, to measure the extent of drug use and to trace the interaction of substance use and the development of psychiatric disorders. ${ }^{6}$

Comprehensive care plans need to initially address the basic physiological needs of drug users, eg. their need for accommodation, food, medical care, psychiatric care, employment and financial support, etc. The application of a 'keyworker/keyagency' system in the community would ensure that drug users are linked up with relevant agencies who can provide the support necessary to address these needs. This system would also ensure assertive follow-up of clients who drop out of treatment.

With regard to the treatment of opiate dependency, there is much research into alternatives to methadone for the treatment of opiate withdrawal and dependency and whether programmes should be focused on abstinence or harm reduction. Magura et al in a review of published literature on post discharge outcomes from methadone detoxification programmes, 'abstinence orientated' methadone programs, and methadone maintenance programmes, found that there was a high reinstatement of opioid use and subsequent increased death rates once discontinuing methadone. ${ }^{26}$ The consensus from the National Institutes of Health is that methadone maintenance programmes are the most effective treatment for opiate dependency when coupled with social services, a comprehensive range of psychological treatments and vocational rehabilitation. ${ }^{27}$

\section{Conclusion}

Compared to clients on a methadone maintenance programme, clients who are on a harm minimisation programme have a poorer perception of their quality of life, as indicated by a higher psychological morbidity and perception of a greater deterioration in health over the previous year. Although being on methadone maintenance led to an improvement in one's perception of 'change in health' over the previous year, psychopathology still existed for clients on a methadone maintenance programme. Comprehensive care programmes need to focus on an individual's psychological, social and physical functioning in order to improve progress in treatment and individuals' perceptions of their quality of life.

References

1. WHO. Constitution of the World Health Organisation. World Health Organisation. Handbook of basic documents, 5th ed. Geneva; 2-30.

2. Ware JE, Brook RH, Davies-Avery A et al. Conceptualisation and measurement of health of adults in the health insurance study. Volume 1: Model of health and methodology. Santa Monica, CA: Rand Corporation, 1980.

3. Stewart AL, Ware JE. Measuring functioning and well-being: the medical outcomes study approach. Durham, North Carolina: Duke University Press. 1992.

4. Torrens M, San L, Martinez A et al. Use of the Nottingham Health Profile for measuring health status of patients in methadone maintenance treatment Addiction 1997; 92(6): 707-16

5. Ryan C, White JM. Health status at entry to methadone maintenance treatment using the SF-36 survey questionnaire. Addiction 1996; 91(1): 39-45.

6. Marsden J, Gossop M, Stewart D et al. Psychiatric symptoms among clients seeking treatment for drug dependence: Intake data from the National Treatment Outcome Research Study. Br J Psychiatry 2000; 176: 285-9.

7. Regier DA, Farmer ME, Rae DS et al. Comorbidity of mental disorders with alcohol and other drug abuse: results from the epidemiologic catchment area (ECA) study. JAMA 1990; 264: 2511-8

8. Rounsaville BJ, Kleber HD. Untreated opiates addicts: how do they differ from those seeking treatment? Arch Gen Psych 1985; 42: 1072-7.

9. Williams H, O'Connor JJ, Kinsella A. Depressive symptoms in opiate addicts on methadone maintenance. Ir J Psychol Med 1990; 7: 45-6.

10. Jenkinson C, Layte R, Wright L et al. The UK SF-36: An analysis and interpretation manual. A guide to health status measurement with particular reference to the Short Form 36 Health Survey. Health Services Research Unit, University of Oxford, 1996

11. Jenkinson C, Wright L, Coulter A. Criterion validity and reliability of the SF-36 in a population sample. Quality of Life Research 1994; 3: 7-12

12. Ware JE. SF-36 Health Survey Manual and Interpretation Guide. Boston MA: Nimrod Press, 1993.

13. Blake C. Codd MB, O’Meara YM. The Short Form 36 (SF-36) Health Survey: normative data for an Irish population. Ir J Med Sci 2000; 169(3): 195200.

14. SF-36TM Health Survey, Copyright (C) 1992 Medical Outcomes Trust. All Rights Reserved. Reproduced with permission of the Medical Outcomes Trust. 15. Zigmond AS, Snaith RP. Hospital Anxiety and Depression Scale. Acta 15. Zigmond AS, Snaith RP. Hospit
Psychiatr Scand 1983; 67: 361-70.

Psychiatr Scand 1983; 67: 361-70.
16. Agresti A. Categorical Data Analysis. Wiley: New York. 1990.

17. Schottenfeld R, Carroll K, Rounsaville B. Comorbid psychiatric disorders and cocaine abuse. (Review) NIDA Res Monogr 1993; 135: 31-47.

18. Milby JB, Sims MK, Khuder S et al. Psychiatric comorbidity: prevalence in methadone maintenance treatment. Am J Drug Alcohol Abuse 1996; 22(1): 95 107.

19. Darke S, Swift W, Hall W. Prevalence, severity and correlates of psychological morbidity among methadone maintenance clients. Addiction 1994; 89(2): 211-7.

20. McLelland AT, Luborsky L, Woody GE et al. Predicting response to alcohol and drug abuse treatments. Arch Gen Psych 1983; 40: 620-5.

21. Hawton K, Fagg J, Platt S. Factors associated with suicide after parasuicide in young people. BMJ 1993; 306: 1641-4.

22. Hall W, Farrell M. Comorbidity of mental disorders with substance misuse. Br J Psych 1997; 171: 4-5.

23. Neeleman J, Farrell M. Suicide and substance misuse. Br J Psych 1997; 171: 303-4.

24. Deykin EY, Buka SL. Suicidal ideation and attempts among chemically 24. Deykin EY, Buka SL. Suicidal ideation and attempts a
dependent adolescents. Am J Public Health 1994; 84: 634-9.

25. Shaffer D, Gould MS, Fischer P et al. Psychiatric diagnosis in child and
depentent a adolescent suicide. Arch Gen Psych 1996; 52: 339-48.

26. Magura S, Rosenblum A. Leaving methadone treatment: lessons learned, lessons forgotten, lessons ignored. (Review) Mount Sinai J Med 2001; 68(1) 62-74.

27. National consensus development panel on effective medical treatment of opiate addiction ( $\mathrm{NiH}$ consensus conference]. Effective medical treatment of opiate addiction. JAMA 1998; 280(22): 1936-43. 